\title{
A Case with Rectal Cancer Relapses After Clinical Complete Remission Following Neoadjuvant Chemoradiotherapy
}

This article was published in the following Dove Press journal: Cancer Management and Research

\author{
Ping-Bao Zhang ${ }^{1,2, *}$ \\ Zi-Li Huang ${ }^{3, *}$ \\ Jia-Bei $\mathrm{Li}^{\prime}$ \\ Xiu-Yan Huang \\ 'Department of General Surgery, \\ Shanghai Jiao Tong University Affiliated \\ Sixth People's Hospital, Shanghai 200233, \\ People's Republic of China; ${ }^{2}$ Department \\ of Urinary Surgery, Affiliated Hospital of \\ Nantong University, Nantong 22602I, \\ People's Republic of China; ${ }^{3}$ Department \\ of Radiology, The Central Hospital of \\ Shanghai Xuhui District, Shanghai \\ 20003I, People's Republic of China
}

*These authors contributed equally to this work
Correspondence: Xiu-Yan Huang Department of General Surgery, Shanghai Jiao Tong University Affiliated Sixth People's Hospital, 600 Yi Shan Road, Shanghai 200233, People's Republic of China

Tel/Fax +86-2I-6470I36I

Email xyhuangIII9@I63.com

\begin{abstract}
Despite advancements in diagnosis and therapy, relapse of rectal cancer after clinical complete remission (cCR) remains a frequent event. The key factors influencing the treatment strategy for the management of patients achieving cCR following neoadjuvant chemoradiotherapy (Neo-CRT) remain to be identified. We present the case of a 64-year-old man with rectal cancer. The patient was initially admitted to the hospital in September 2011 with a 3-month history of change in his stools. Following his re-hospitalization in November 2011, a biopsy specimen of the neoplasm suggested the presence of rectal adenocarcinoma; laboratory investigations also revealed elevated levels of carcinoembryonic antigens (CEA; carbohydrate antigen 199) in the serum. Subsequently, the patient received Neo-CRT, as well as symptomatic and supportive treatment. The level of serum CEA returned to normal, without signs of swollen lymph nodes in the pelvic cavity. The patient was diagnosed with rectal cancer based on the elevated level of serum CEA, colonoscopy, and contrastenhanced magnetic resonance imaging. He relapsed 4 months after cCR following Neo-CRT and underwent laparoscopic Miles' surgery in April 2013. The relapse may have been mainly attributed to residual tumor cells. This case report and literature review may contribute to the clinical recognition of treatment for patients with rectal cancer achieving cCR following Neo-CRT.
\end{abstract}

Keywords: rectal cancer, neoadjuvant chemoradiotherapy, Neo-CRT, clinical complete remission, cCR

\section{Introduction}

Colorectal cancer (CRC) is one of the most common malignancies in the United States. ${ }^{1}$ The introduction of new and effective chemotherapeutic substances and biologics during the past decade has significantly improved the systemic treatment of CRC patients. ${ }^{2}$ Neoadjuvant chemoradiotherapy (Neo-CRT) is standard treatment for stage II-III rectal cancer, which consists of chemotherapy and radiotherapy aiming at reducing locoregional relapse. ${ }^{3}$

Evidence exists to support both surgical and nonoperative observational approaches to the management of patients with distal rectal cancer who achieve a complete response following Neo-CRT. ${ }^{4}$ This report describes one rectal cancer patient, achieving clinical complete remission (cCR) following Neo-CRT, got relapse 4 months later. Approaches of surgery are also discussed.

\section{Case Report}

A 64-year-old male, without smoking, alcohol addiction, or any other specific underlying disease histories, was initially admitted to the hospital in September 2011 with 
a 3-month history of change in his stools. Colonoscopy showed an ulcer-like neoplasm situated $6 \mathrm{~cm}$ from the anal margin with a little bleeding, covering half of the lumen (Figure 1A). A biopsy specimen of the neoplasm suggested high-grade intraepithelial neoplasia in rectal mucosa. The patient was re-hospitalized in November 2011. The reexamination of colonoscopy showed an ulcer type neoplasm on the dentate line with erosion, which was brittle and subjected to hemorrhage, covering half of the lumen (Figure 1B). A biopsy specimen of the neoplasm suggested rectal adenocarcinoma. Laboratory investigations disclosed elevated levels of carcinoembryonic antigens (CEA; $12.2 \mathrm{ng} /$ $\mathrm{mL}$; normal range: $0.0-5.0 \mathrm{ng} / \mathrm{mL})$ and CA199 $(38.59 \mathrm{U} / \mathrm{mL}$; normal range: $0.00-37.00 \mathrm{U} / \mathrm{mL}$ ) in the serum Contrastenhanced MRI showed uneven thickening in rectal wall which consisted heterogeneous enhancement in enhanced scanning. The patient was then given radiotherapy with conventional fractionation as follows (Treatment region A: rectum, 200cGy per day; five fractions per week, total dose, $5000 \mathrm{cGy} / 25$ fractions, and treatment region B: pelvic cavity, 180cGy per day; five fractions per week, total dose, 4500 cGy/25 fractions). In February 2012, he had a reexamination of colonoscopy, which showed a $2 \times 2 \mathrm{~cm} 2$ ulcer on distal rectal wall (near the dentate line) with white tongue coating (Figure 1C). A biopsy specimen of the ulcer suggested rectal mucosal erosion. Contrast-enhanced MRI showed slight incrassation on lower rectal wall, no significant abnormal enhancement was found. Afterward, the patient started chemotherapy which is given in 3 week cycles and consists of capecitabine $1250 \mathrm{mg} / \mathrm{m}^{2}$ twice a day, day 1-14 combined with oxaliplatin $130 \mathrm{mg} / \mathrm{m}^{2}$ once every 3 weeks, 5 cycles of chemotherapy in total. Treatment-related toxicity is monitored through the procedure and during which the patient was also receiving symptomatic and supportive treatment. The patient was re-examined in April 2012, his serum level of carcinoembryonic antigens came out to be CEA, 1.70
A

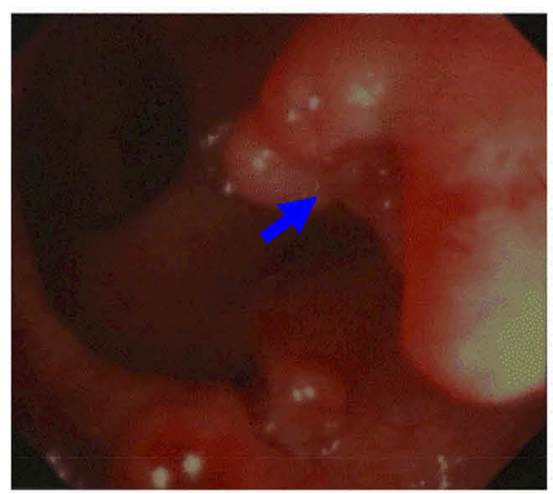

C

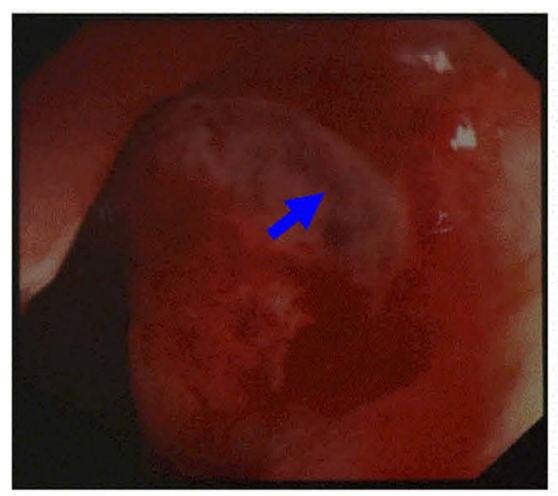

B

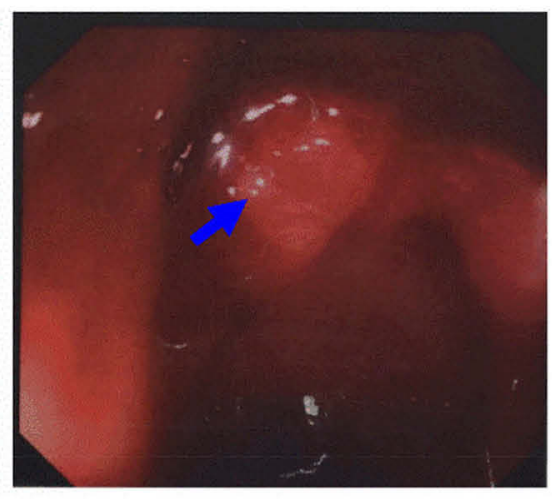

D

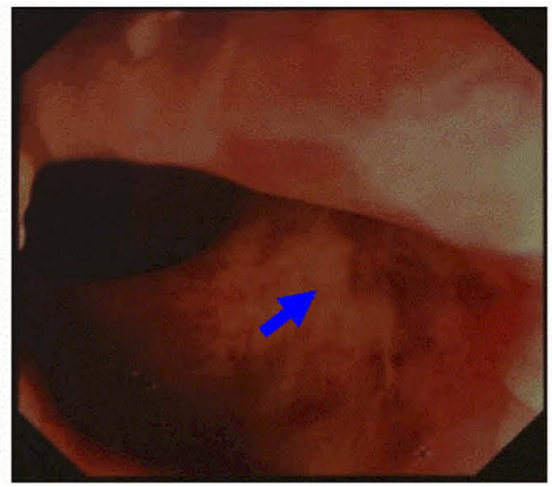

$\mathbf{E}$

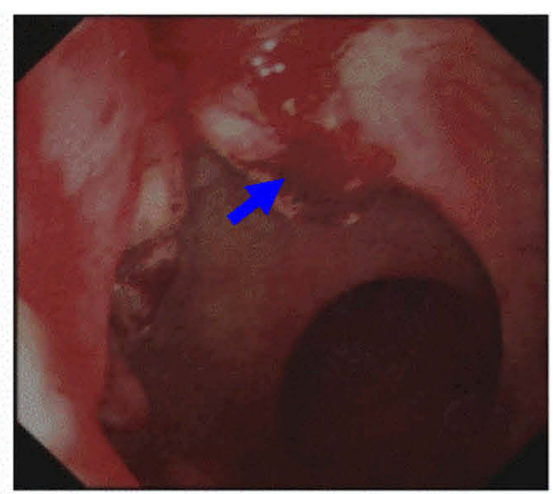

Figure I Results of colonoscopy. (A) Colonoscopy showed an ulcer-like neoplasm situated $6 \mathrm{~cm}$ from the anal margin with a little bleeding, covering half of the lumen in September 2011 (arrows). (B) The re-examination of colonoscopy showed an ulcer type neoplasm on dentate line with erosion, which was brittle and subjected to hemorrhage, covering half of the lumen in November 201 I (arrows). (C) In February 20I2, the re-examination of colonoscopy, which showed a $2 \times 2 \mathrm{~cm} 2$ ulcer on distal rectal wall (near the dentate line) with white tongue coating (arrows). (D) In August 20I2, the colonoscopy showed a scar with smooth surface on distal rectal wall (near the dentate line, arrows). (E) In March 2013, the colonoscopy showed a $2 \mathrm{~cm} \times 4 \mathrm{~cm}$ neoplasm on the dentate line with erosion, which was brittle and subjected to hemorrhage (arrows). 
ng/mL; normal range: 0.0-5.0 ng/mL, CA199, $14.25 \mathrm{U} / \mathrm{mL}$; normal range: 0.00-37.00 U/mL. In August 2012, a contrastenhanced MRI showed a high T2 signal slight incrassation on lower rectal wall, CEA,2.71 ng/mL; normal range: 0.0-5.0 ng/mL, CA199, $11.90 \mathrm{U} / \mathrm{mL}$; normal range: 0.00-37.00 U/ $\mathrm{mL}$, and the colonoscopy showed a scar with smooth surface on distal rectal wall (near the dentate line) (Figure 1D). In October 2012, another contrast-enhanced MRI showed a high T2 signal $1.2 \times 1.2 \mathrm{~cm}$ tubercle with enhanced edge on the right wall of lower rectum (near the anal canal), no signs of swollen lymph node was found in pelvic cavity. In March 2013, the laboratory investigation came out to be CEA, $8.65 \mathrm{ng} / \mathrm{mL}$; normal range: $0.0-5.0 \mathrm{ng} / \mathrm{mL}$, CA199, 27.21 U/mL; normal range: $0.00-37.00 \mathrm{U} / \mathrm{mL}$, the colonoscopy showed a $2 \mathrm{~cm} \times 4 \mathrm{~cm}$ neoplasm on dentate line with erosion, which was brittle and subjected to hemorrhage (Figure 1E). A biopsy of the resected specimen suggested villous adenoma, suspecting canceration, and contrastenhanced MRI showed a high T2 signal $1.5 \mathrm{~cm} \times 1.5 \mathrm{~cm}$ tubercle with an enhanced edge on the right wall of the lower rectum (near the anal canal), no signs of swollen lymph node found in the pelvic cavity.

According to the history, the patient should be diagnosed as rectal cancer relapsed 4 months after cCR under Neo-CRT. There was no sign of cancer metastasis observed before the surgery. The patient received Laparoscopic Mile's surgery in April 2013, during which no metastatic nodules was observed on abdominal viscera, and the tumor was located below the pelvic peritoneal reflex. The surgery dissociated the sigmoid colon and the rectum, then closed the incision with EC60 (made in Shanghai jmu medical device co. LTD, Shanghai, China) and made sigmoid flexure fistula at the leftside abdomen. The tumor specimen consisted of the anus and rectum (length: $13 \mathrm{~cm}$ ). The tumor located in the rectum measured $1.5 \times 1.2 \mathrm{~cm}, 5.5 \mathrm{~cm}$ from the upper band, and $2.5 \mathrm{~cm}$ from the dentate line. Gross morphological analysis showed ulcer infiltration type, growing around a quarter of the rectal wall. The tumor infiltrated peri-nerve tissue, but no sign of invasion of lymphatic vessel or blood vessel observed. No sign of tumor infiltration on both upper and lower bands. Lymph node metastases: all four mesenteric lymph nodes were clear, and the immune response $(\mathrm{SH}$, $\mathrm{PH}, \mathrm{GH}$ ) of the lymph nodes was all positive. The precancerous interstitial reaction consisted of lymphocyte, plasmacyte, histocyte reaction and fibrosis proliferation reaction, and the non-tumor area reaction came out to be chronic mucosa tissue inflammation. Microscopic findings of the rectum mass showed tubular adenocarcinoma (stage II-III, Figure 2A). The immunoenzyme labeled Ki-67 test showed 55\% of the tumor tissue was positive (Figure $2 \mathrm{~B}$ ). The postoperative follow-up showed the patient was recovering very well. Table 1 shows the patient's disease stage.

\section{Discussion}

$\mathrm{CRC}$ is the third most commonly diagnosed cancer among both men and women, with over 135,400 new colorectal cancer cases and 50,000 deaths estimated to have occurred in the United States in 2017. ${ }^{1,5}$ The highest incidence rates are found in Australia and New Zealand, Europe, and North America, whereas the lowest rates are found in Africa and South-Central Asia. Rates are substantially higher in males than in females. ${ }^{6}$ Neo-CRT is a standard treatment for stage II-III rectal cancer. ${ }^{7}$ A proportion of patients, who receive preoperative chemoradiation for locally advanced (T3, T4, NX) rectal cancer, achieve a $\mathrm{cCR}$ and a pathologic complete remission ( $\mathrm{pCR}$ ) in the
A

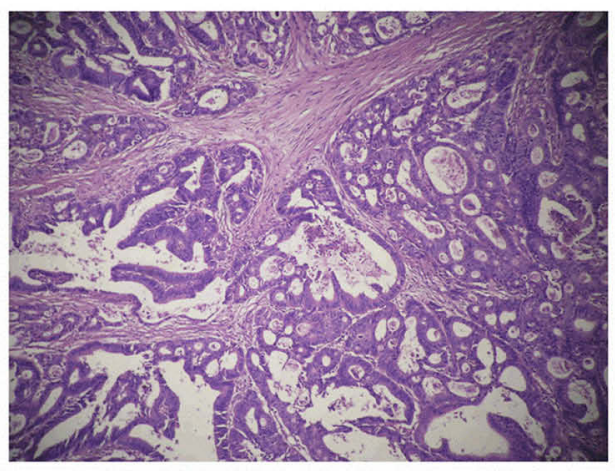

B

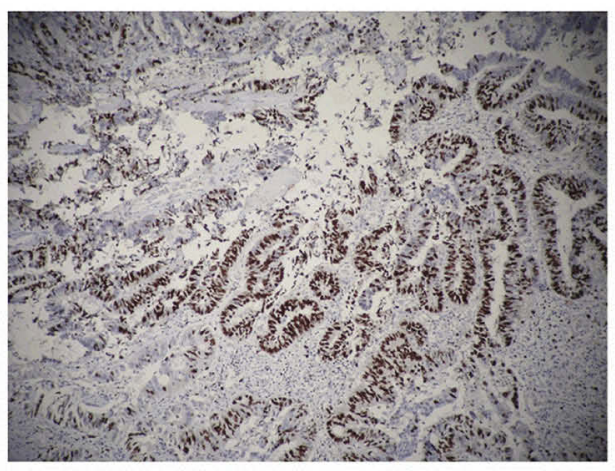

Figure 2 Microscopic findings of the rectum mass. (A) Results showed tubular adenocarcinoma (H-E, original magnification, $\times 100)$. (B) The immunoenzyme labeled Ki-67 test showed $55 \%$ of the tumor tissue was positive $(\mathrm{IH}$, original magnification, $\times 100)$. 
Table I The Disease Stage of the Patient

\begin{tabular}{|l|l|l|}
\hline Time & Treat & Stage \\
\hline 2011.9 & Endoscopic follow-up & PTINOM0 \\
2011.11 & Endoscopic follow-up & cT3NxM0 \\
2012.2 & Radiotherapy & yPTxN0M0 \\
2012.8 & Chemotherapy & yPTxN0M0 \\
2012.12 & Endoscopic follow-up & rT3NxM0 \\
2013.3 & Surgery & rT4NOM0 \\
\hline
\end{tabular}

region of $15 \%$ to $30 \%{ }^{8}$ digital rectal examination (DRE) is only able to identify a small proportion of patients who actually achieve a pCR, and only $25 \%$ to $50 \%$ of patients achieving a cCR are confirmed as a pCR at subsequent surgery. ${ }^{9}$ Complete clinical response represents the patient is disease-free according to the colonoscopy biopsy after Neo-CRT, while a pathologic complete response patient is diagnosed by observing cancer cell in the specimen cut during the surgery after Neo-CRT. Patients with rectal cancer receiving preoperative chemoradiotherapy can experience late failures. ${ }^{10,11}$

The predicted risk of local recurrence in rectal cancer is dependent on the depth of tumor invasion, lymphovascular involvement, tumor differentiation and lymph node involvement. These factors also influence the risk of developing metastatic disease. A study showed that $17 \%$ of pCR patients with identifiable nodal involvement were predicted to be at high risk of developing locally recurrent disease, and approximately $30-50 \%$ of those were expected to develop simultaneous disseminated disease. ${ }^{12}$ It suggests that, for cCR patients, there could be tumor cells remaining after NeoCRT. And referring to a recent review about 502 rectal cancer cases, the mean lymph node yield for proctectomy specimens without Neo-CRT was 13 , with a median of 10 lymph nodes.
In contrast, specimens after neoadjuvant therapy (NAT) had a mean lymph node yield of 9 , with a median of 7 lymph nodes. ${ }^{13}$ Comparing to this case, considering the patient achieved cCR following Neo-CRT, and all four mesenteric lymph nodes were clear; the key to his relapse could be the remaining of the cancer cells. So it is worth taking into consideration how to treat patients who have already achieved cCR after Neo-CRT. Or, on the basis of the existing Smith, ${ }^{14}$ Habr, ${ }^{15}$ and Maas ${ }^{16}$ standards, we may need to develop a better assessment so that the assessment of cCR is more accurate. The major view thinks it impossible to find out whether a cCR patient has achieved $\mathrm{pCR}$, so cCR patients have to take the total mesorectal excision (TME). But according to studies from University of Sao Paulo, recurrence and cancer-related mortality rates showed no statistical difference between stage $\mathrm{p} 0$ and stage $\mathrm{c} 0(\mathrm{P}=0.2) .{ }^{17}$ According to some studies, long-term oncological outcomes after a cCR and deferred strategy are favorable. ${ }^{18,19}$ Moreover, combined upfront chemoradiotherapy was associated with tolerable and acceptable side effects. A significant number of patients had complete disappearance of their tumors (30.5\%) within a median follow-up of 36 months. ${ }^{20}$ Therefore, it was suggested that patient achieving cCR after Neo-CRT should be subjected to long-term follow-up monitoring of the CEA and CA199 levels. It seems that CEA was more sensitive than CA199 in this case (Figure 3). Ulrik Wallin et al have demonstrated an association between low pretreatment CEA levels, interruption in chemoradiation therapy, and pathologic complete response in nonsmoking patients treated with Neo-CRT for locally advanced rectal cancer. ${ }^{21}$ In addition to CEA, focal adhesion kinase (FAK) and Ku70/Ku80 were also studied as predictors of tumor response and risk factor for recurrence after Neo-CRT in rectal cancer. ${ }^{22,23}$ In this setting, the selection of a single or a combination of

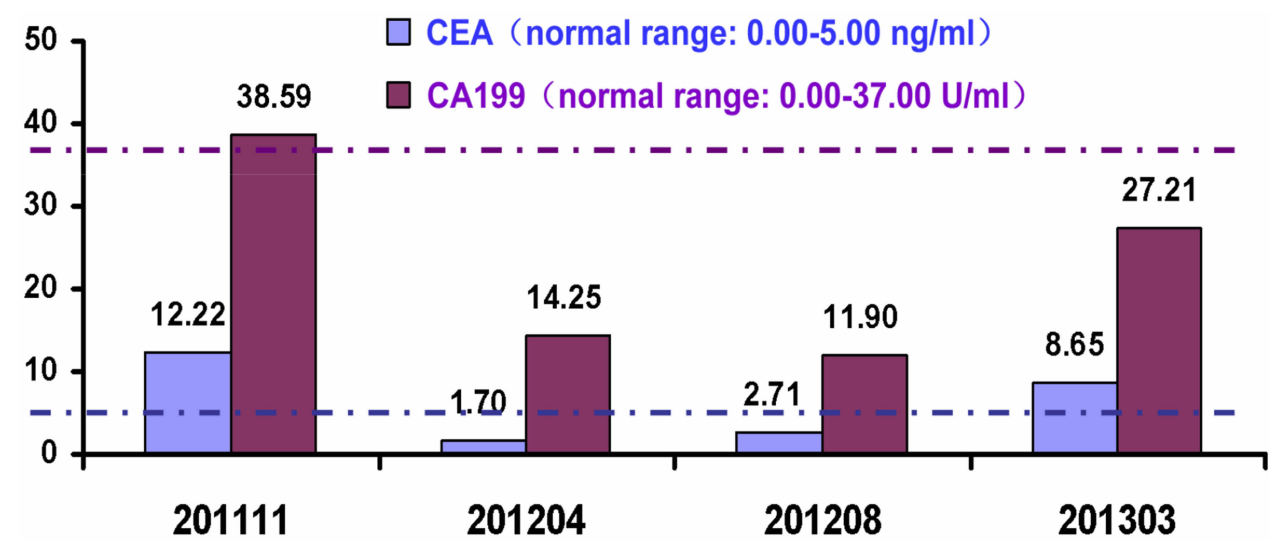

Figure 3 Follow-up with monitoring CEA and CAI99. CEA seemed more sensitive than CAI99 in this case. 
multiple biological markers for the accurate prediction of cCR can determine the sensitive population and allow precise treatment of different individuals. There are also scholars honoring minimally invasive surgeries rather than ordinary ones. There is already a trend to cure cCR patient with rectal cancer using minimally invasive laparoscopic surgeries. It is also confirmed by the study about surgery versus open resection for mid-low rectal cancer. There was no statistical difference in local recurrence rate $(9.1 \%$ vs $6.4 \%$; log-rank $=$ $0.432 ; \mathrm{P}=0.511)$ and distant recurrence rate $(19.7 \%$ vs $15.5 \%$; log-rank $=0.505 ; \mathrm{P}=0.477$ ) between the laparoscopic and open groups in 5 years. ${ }^{24}$ That is why laparoscopic surgery is becoming the standard procedure of the mid-low rectal cancer. Recently, transanal total mesorectal excision (TaTME) has emerged as a focused area of laparoscopic surgery that is becoming a safe and feasible approach in the rectal surgery. ${ }^{25}$ In this case, the patient was unwilling to take the surgery by the time achieved cCR after Neo-CRT, but he changed his mind when the relapse was observed and confirmed during the follow-up, and eventually recovered well after the surgery. We can infer from this case that patients achieved cCR after Neo-CRT should keep followup constantly. More frequent follow-up visits may contribute to an early diagnosis of cancer. ${ }^{26}$ Operation will be performed when the relapse is confirmed. However, minimally invasive surgery should be recommended for a patient receiving abdominoperineal resection.

\section{Ethics Statement}

This study was approved by the Research Ethics Committee of Shanghai Jiaotong University affiliated Sixth People's Hospital, and informed consent was obtained from the patient. All specimens were collected in the operating room immediately ( $\leq 15 \mathrm{~min}$ ) after tissue removal and were snap-frozen in liquid nitrogen and stored at $-80^{\circ} \mathrm{C}$. All methods were performed in accordance with the relevant guidelines and regulations. Participants gave their written informed consent for the materials to appear in publications without limit on the duration of publication.

\section{Acknowledgments}

This study was supported by grants from the medicalengineering cross fund of Shanghai Jiao Tong University (No. YG2017MS13), the pre-research fund of Shanghai Sixth People's Hospital (LYZY-0229), the International Foundation of Translational Medicine for Abroad Scholars and Students, US and China (No. UCTMP2015-
03C001), and the National Natural Science Foundation of China (No. 81272401).

\section{Disclosure}

The authors report no conflicts of interest in this work.

\section{References}

1. Siegel RL, Miller KD, Fedewa SA, et al. Colorectal cancer statistics, 2017. CA Cancer J Clin. 2017;67(3):177-193. doi:10.3322/caac.21395

2. Pohl M, Schmiegel W. Colorectal cancer - personalized, stageadjusted tumour therapy. Dtsch Med Wochenschr. 2013;138 (36): 1790-1795. doi:10.1055/s-0033-1343343

3. Xiao J, Tan Y, Li W, et al. Tumor volume reduction rate is superior to RECIST for predicting the pathological response of rectal cancer treated with neoadjuvant chemoradiation: results from a prospective study. Oncol Lett. 2015;9(6):2680-2686. doi:10.3892/ol.2015.3101

4. Dedemadi G, Wexner SD. Complete response after neoadjuvant therapy in rectal cancer: to operate or not to operate? Digestive Dis. 2012;30(Suppl 2):109-117. doi:10.1159/000342039

5. Siegel RL, Miller KD, Jemal A. Cancer statistics, 2017. CA Cancer J Clin. 2017;67(1):7-30. doi:10.3322/caac.21387

6. Jemal A, Bray F, Center MM, Ferlay J, Ward E, Forman D. Global cancer statistics. CA Cancer J Clin. 2011;61(2):69-90. doi:10.3322/ caac. 20107

7. Martin LK, Bekaii-Saab T. Optimizing neoadjuvant therapy for rectal cancer with oxaliplatin. J Natl Compr Cancer Net. 2013;11(3):298-307. doi:10.6004/jnccn.2013.0041

8. De Felice F, Izzo L, Musio D, et al. Clinical predictive factors of pathologic complete response in locally advanced rectal cancer. Oncotarget. 2016;7(22):33374-33380. doi:10.18632/oncotarget.v7i22

9. Glynne-Jones R, Wallace M, Livingstone JI, Meyrick-Thomas J. Complete clinical response after preoperative chemoradiation in rectal cancer: is a "wait and see" policy justified? Dis Colon Rectum. 2008;51 (1):10-19; discussion 19-20. doi:10.1007/s10350-007-9080-8

10. Valentini V, Minsky BD. Tumor regression grading in rectal cancer: is it time to move forward? J Clin Oncol. 2014;32(15):1534-1536. doi:10.1200/JCO.2014.55.4766

11. Tan Y, Fu D, Li D, et al. Predictors and risk factors of pathologic complete response following neoadjuvant chemoradiotherapy for rectal cancer: a population-based analysis. Front Oncol. 2019;9:497. doi: $10.3389 /$ fonc. 2019.00497

12. Hughes R, Glynne-Jones R, Grainger J, et al. Can pathological complete response in the primary tumour following pre-operative pelvic chemoradiotherapy for T3-T4 rectal cancer predict for sterilisation of pelvic lymph nodes, a low risk of local recurrence and the appropriateness of local excision? Int J Colorectal Dis. 2006;21 (1):11-17. doi:10.1007/s00384-005-0749-y

13. Amajoyi R, Lee Y, Recio PJ, Kondylis PD. Neoadjuvant therapy for rectal cancer decreases the number of lymph nodes harvested in operative specimens. Am J Surg. 2013;205(3):289-292. doi:10.1016/j.amjsurg. 2012.10.020

14. Smith JD, Ruby JA, Goodman KA, et al. Nonoperative management of rectal cancer with complete clinical response after neoadjuvant therapy. Ann Surg. 2012;256(6):965-972. doi:10.1097/SLA.0b013e3182759f1c

15. Habr-Gama A, Perez RO, Wynn G, Marks J, Kessler H, GamaRodrigues J. Complete clinical response after neoadjuvant chemoradiation therapy for distal rectal cancer: characterization of clinical and endoscopic findings for standardization. Dis Colon Rectum. 2010;53(12):1692-1698. doi:10.1007/DCR.0b013e3181f42b89

16. Maas M, Beets-Tan RG, Lambregts DM, et al. Wait-and-see policy for clinical complete responders after chemoradiation for rectal cancer. J Clin Oncol. 2011;29(35):4633-4640. doi:10.1200/JCO. 2011.37.7176 
17. Habr-Gama A, Perez RO, Nadalin W, et al. Long-term results of preoperative chemoradiation for distal rectal cancer correlation between final stage and survival. J Gastrointest Surg. 2005;9 (1):90-99. doi:10.1016/j.gassur.2004.10.010

18. Gani C, Kirschniak A, Zips D. Watchful waiting after radiochemotherapy in rectal cancer: when is it feasible? Visc Med. 2019;35 (2):119-123. doi:10.1159/000499167

19. Couch DG, Hemingway DM. Complete radiotherapy response in rectal cancer: a review of the evidence. World $J$ Gastroenterol. 2016;22(2):467-470. doi:10.3748/wjg.v22.i2.467

20. Habr-Gama A, de Souza PM, Ribeiro U Jr., et al. Low rectal cancer: impact of radiation and chemotherapy on surgical treatment. Dis Colon Rectum. 1998;41(9):1087-1096. doi:10.1007/BF02239429

21. Wallin U, Rothenberger D, Lowry A, Luepker R, Mellgren A. CEA a predictor for pathologic complete response after neoadjuvant therapy for rectal cancer. Dis Colon Rectum. 2013;56(7):859-868. doi:10.1097/DCR.0b013e31828e5a72

22. Gomez Del Pulgar T, Cebrian A, Fernandez-Acenero MJ, et al. Focal adhesion kinase: predictor of tumour response and risk factor for recurrence after neoadjuvant chemoradiation in rectal cancer. $J$ Cell Mol Med. 2016;20(9):1729-1736. doi:10.1111/jcmm.12879
23. Pucci S, Polidoro C, Joubert A, et al. Ku70, Ku80, sClusterin: a "cluster" of predicting factors for neoadjuvant chemoradiotherapy treatment response in patients affected by locally advanced rectal cancer. Int J Radiat Oncol. 2016.

24. Li S, Chi P, Lin H, Lu X, Huang Y. Long-term outcomes of laparoscopic surgery versus open resection for middle and lower rectal cancer: an NTCLES study. Surg Endosc. 2011;25(10):3175-3182. doi:10.1007/s00464-011-1683-4

25. Chen CC, Lai YL, Jiang JK, et al. Transanal total mesorectal excision versus laparoscopic surgery for rectal cancer receiving neoadjuvant chemoradiation: a matched case-control study. Ann Surg Oncol. 2016;23(4):1169-1176. doi:10.1245/s10434-015-4997-y

26. Cai Y, Li Z, Gu X, Fang Y, Xiang J, Chen Z. Prognostic factors associated with locally recurrent rectal cancer following primary surgery (Review). Oncol Lett. 2014;7(1):10-16. doi:10.3892/ ol.2013.1640

\section{Publish your work in this journal}

Cancer Management and Research is an international, peer-reviewed open access journal focusing on cancer research and the optimal use of preventative and integrated treatment interventions to achieve improved outcomes, enhanced survival and quality of life for the cancer patient.
The manuscript management system is completely online and includes a very quick and fair peer-review system, which is all easy to use. Visit http://www.dovepress.com/testimonials.php to read real quotes from published authors. 